\title{
Newborn screening for Tyrosinemia type 1 using succinylacetone - a systematic review of test accuracy
}

\author{
Chris Stinton', Julia Geppert ${ }^{1}$, Karoline Freeman ${ }^{1}$, Aileen Clarke ${ }^{1}$, Samantha Johnson ${ }^{2}$, Hannah Fraser ${ }^{1}$,
} Paul Sutcliffe ${ }^{1}$ and Sian Taylor-Phillips ${ }^{1 *}$

\begin{abstract}
Background: Tyrosinemia type 1 is an autosomal recessive disorder of amino acid metabolism. Without treatment, death in childhood is common. Treatment with nitisinone and dietary restrictions are associated with improved outcomes; some studies suggest better outcomes when treatment begins at an asymptomatic stage. Newborn screening allows for earlier identification, but there is uncertainty regarding the test accuracy of the current method: succinylacetone measurement in dried blood spots using tandem mass spectrometry.
\end{abstract}

Methods: We conducted a systematic review of literature published up to January 2016. Two reviewers independently assessed titles, abstracts, full texts, and conducted quality appraisals. A single reviewer extracted data, which was checked by a second reviewer.

Results: Ten studies provided test accuracy data: five studies reporting screening experiences and five case-control studies. Sensitivity (29 cases in total) and specificity (34,403 controls in total) were 100\% in the case-control studies, but could not be calculated in the studies reporting screening experiences due to a lack of follow-up of screen-negative babies. Positive predictive values in the screening experience studies ranged from 66.7\% (2 true positive cases, 1 false positive case from $~ 500,000$ people screened) to 100\% ( 8 true positive cases from 856,671 people screened); negative predictive values could not be calculated. Positive and negative predictive values cannot be calculated from case-control studies.

Conclusions: Screening for Tyrosinemia type 1 using tandem mass spectrometry measurement of succinylacetone from dried blood spots appears to be promising. Confirmation of test accuracy data should be obtained from studies that include a two-year follow-up of individuals who screen negative.

Keywords: Systematic review, Tyrosinemia, Test accuracy, Succinylacetone, Inborn errors of metabolism, Newborn blood spot screening, Tandem mass spectrometry

\section{Background}

Tyrosinemia type 1 (TYR1), also known as fumarylacetoacetase deficiency (Enzyme Commission Number 3.7.1.2), is an autosomal recessive disorder of amino acid metabolism. It is caused by a deficiency in the activity of fumarylacetoacetic hydrolase, the final enzyme in the tyrosine degradation pathway, which leads to a toxic build-up of fumarylacetoacetate, maleylacetoacetate, and succinylacetone (SUAC) [1]. TYR1 is characterised by

\footnotetext{
* Correspondence: S.Taylor-Phillips@warwick.ac.uk

${ }^{1}$ Warwick Medical School, University of Warwick, Coventry CV4 7AL, England Full list of author information is available at the end of the article
}

progressive liver, kidney, and neurological disease [2]. Acute (presenting before six months of age), sub-acute (presenting between six and 12 months) and chronic (presenting after one year) forms of the disease have been described [2]. Without treatment, the prognosis for individuals with TYR1 is poor, with high levels of death during childhood due to liver failure, recurrent bleeding, hepatocellular carcinoma, and porphyria-like syndrome with respiratory failure [3]. However, treatment with nitisinone and dietary restrictions are associated with reductions in morbidity and mortality [4-6]; liver transplantation is indicated if these treatment fail or if 
hepatocellular carcinoma develops [2]. The incidence of TYR1 is estimated to be approximately 1:100,000 live births, but reported values range from 1:1,846 [7] to 1:781,144 live births [8]. The incidence of TYR1 is higher in Quebec, Canada, possibly due to a founder effect for Tyrosinemia and high gene frequency [7], and in Asian children in the West Midlands of the UK [9], and in North Africa and the Middle East [10], possibly due to parental consanguinity [9].

Screening for TYR1 amongst newborn babies is conducted in many countries around the world. While tyrosine levels have been used as the primary screening marker for TYR1, it is not consistently raised in individuals who have TYR1 [11], and it can be elevated in individuals with other conditions and in unaffected babies $[12,13]$. In 2004, Allard and colleagues developed an alternative method to screen for TYR1 using tandem mass spectrometry (MS/MS) to determine SUAC in dried blood spots (DBS) [14]. A rapid review of literature published up to 2012 reported that "Screening programmes using succinylacetone as a marker have reported $100 \%$ sensitivity and $100 \%$ specificity. However, other studies have reported the identification of false positives." [15]. The aim of the current review was to examine the range of test accuracy indicators (sensitivity, specificity, and predictive values) of succinylacetone measurement in DBS using MS/MS for TYR1 screening using full systematic review methods.

\section{Methods}

\section{Search strategy}

We conducted searches in the following electronic databases: Medline, Medline In-Process \& Other NonIndexed Citations, Embase, Web of Science (All Databases), and the Cochrane Library. We searched using text word and $\mathrm{MeSH}$ terms relating to "Tyrosinemia type 1 OR inborn errors of metabolism", AND "succinylacetone OR DBS OR (tandem mass spectrometry AND neonatal screening)". Full details of the search strategy are provided in Additional file 1: supplement 1. The search was conducted on $26^{\text {th }}$ January 2016. We examined reference lists of included studies and previous reviews. Experts in the field and organisations were contacted for studies not in the public domain.

\section{Eligibility criteria}

We included English language journal articles which investigated screening for TYR1 by MS/MS analysis of SUAC from DBS in newborns. The reference standard was urine testing for SUAC, clinical detection of TYR1 or two-year follow-up. Outcomes included were any reported test accuracy measures from cross-sectional studies, casecontrol studies, or studies reporting screening experiences. We excluded non-human studies, papers not available in
English, letters, editorials, communications, grey literature, conference abstracts, and studies published before 2004 (the year the first paper was published on SUAC measurement in DBS using MS/MS for TYR1) from our review.

\section{Screening and data extraction}

Screening of titles and abstracts of all retrieved records, and subsequently of full texts, was undertaken independently by two reviewers. Data extraction was performed by a single reviewer, with all data extraction forms checked by a second reviewer. Disagreements were resolved by discussion between the two reviewers or further discussion with a third reviewer, leading to a consensus on inclusion/exclusion.

\section{Quality appraisal}

Quality of included studies was assessed independently by two reviewers using the Quality Assessment Tool for Diagnostic Accuracy Studies 2 [QUADAS-2; [16]] which was tailored to the research as recommended. Tailoring of the QUADAS-2 tool included adding a topic-specific signalling question and defining appropriate reference standards and cut-offs for participant exclusions, as well as guidance on how many positive signalling questions are required for an overall positive rating in terms of bias and applicability concerns. (See Additional file 1: supplement 2 for signalling questions and Additional file 1: supplement 3 for guidance notes). Disagreements were resolved by discussion between the two reviewers or through discussion with a third reviewer, leading to a consensus on study quality.

\section{Data summary and synthesis}

Meta-analysis was not possible due to incomplete $2 \times 2$ tables and heterogeneity in study design. Therefore, a narrative synthesis of results is provided.

\section{Results}

\section{Searching, sifting, and sorting}

One thousand two hundred and seventy-five unique records were identified. Seventy six were retained after sifting titles and abstracts. Assessment of full text papers against inclusion/exclusion criteria resulted in ten studies being included in our review; five studies reporting screening experiences [12, 17-20] and five retrospective case-control studies using stored samples from known TYR1 cases [14, 21-24]. All ten papers were identified through electronic database searches. Full details regarding the numbers of studies retained and excluded at each stage of the review is provided in Fig. 1. A list of excluded studies (with reasons) is given in Additional file 1: supplement 4. 


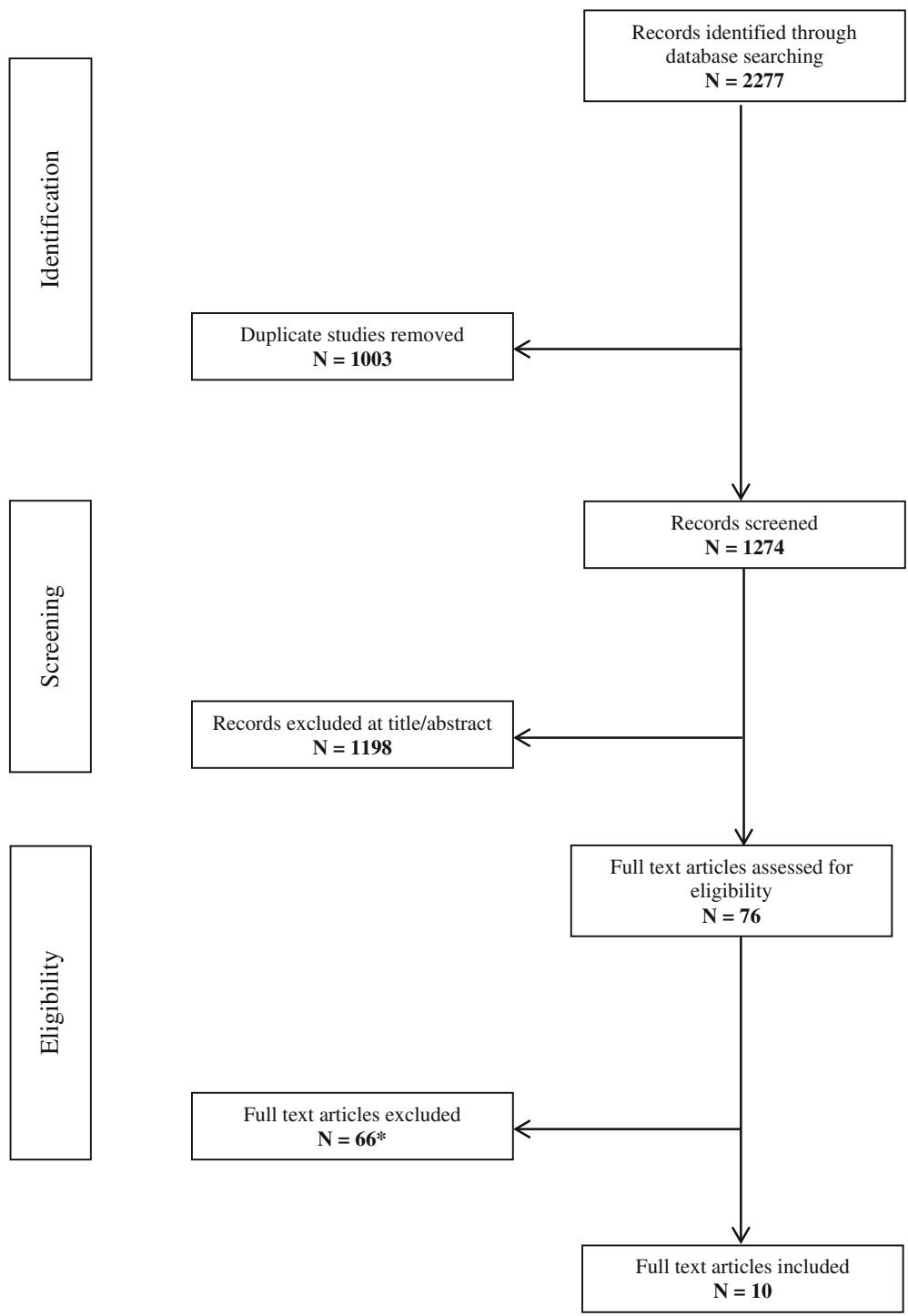

Fig. 1 PRISMA flow diagram of records through the systematic review. *See Additional file 1: supplement 4 for list of excluded studies with reasons

\section{Quality appraisal}

The overall risk of bias and applicability concerns of the included studies are provided in Fig. 2. A summary of the methodological quality for each of the included studies is given in Additional file 1: supplement 5. Risk of bias was considered high in two or more domains in six of ten studies $(60 \%)$ and in one domain in the remaining four studies (40\%). No study was judged as low or unclear risk of bias in all four domains. In the patient selection domain, all five case-control studies

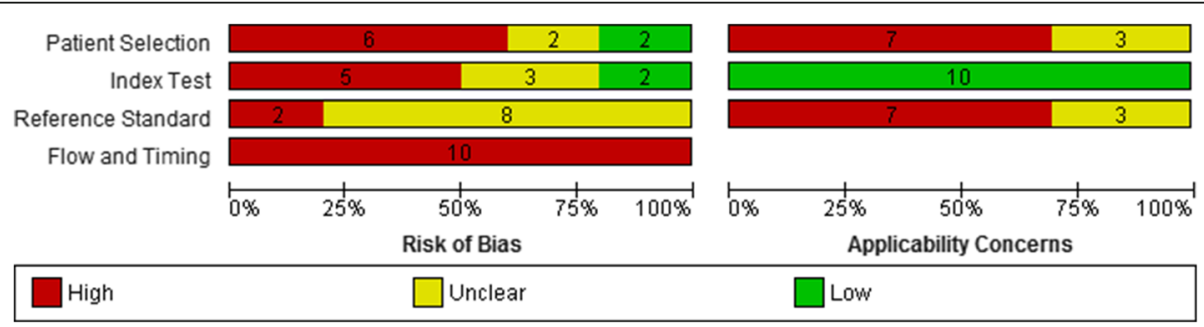

Fig. 2 Risk of bias and applicability concerns graph: review authors' judgements about each domain presented as percentages across included studies 
$[14,21-24]$ were considered to be at a high risk of bias. One study reporting a screening experience [20] was considered to be at a high risk of bias as the study population included screening samples taken from babies that were symptomatic and/or outside the 'newborn' period. There were significant concerns regarding applicability of the research to the UK screening population in seven studies as the incidence of TYR1 was higher than expected in the UK population and/or screened dried blood spot samples were collected before five days or after eight days of life [14, 17-20, 22, 24].

In the index test domain, five studies were judged to have a high risk of bias as the results of the reference test were known when interpreting the index test in all case-control studies and the SUAC threshold was not pre-specified [14, 21-24]. Applicability concerns were low for all ten studies.

In the reference test domain, two studies were judged to be at high risk of bias as case-controls received a screening test (second-tier test measuring SUAC directly or indirectly in babies with elevated tyrosine levels) as a reference standard $[23,24]$. The remainder of the studies had an unclear risk of bias as they did not report the method of diagnosis, or did not report sufficient information to allow a judgement to be made [12, 14, 17-22]. Applicability concerns were high in seven studies as babies that screened negative in studies reporting screening experiences or were used as controls did not receive an appropriate reference standard, i.e. diagnostic testing or clinical follow-up for at least two years [12, 17-20, 23, 24].

In the flow and timing domain, all ten studies were considered to be at high risk of bias. The reasons for this were that the reference standards used to confirm TYR1 status for screen-positives and screen negatives (or cases and controls) were not the same, follow-up of those people who screened-negative was not defined or not conducted, and losses to follow-up were not reported $[12,14,17-24]$.

\section{Characteristics of included studies}

Included studies are summarised in table 1 and Additional file 1: supplement 6 . There were ten studies. Five studies reported experiences of newborn screening programmes [12, 17-20]. Data were given for screening periods ranging from 16 weeks [19] to four years one month [20]; the number of analysed screening samples ranged from 61,344, which included two cases [19], to 518,687 , which included three cases [20]. Five papers reported on case-control studies [14, 21-24] conducted over periods between one [21] and five [22] months; the number of screening samples ranged from $~ 1000$, which included six cases [21], to 13,532, which included 11 cases [24].

\section{Description of screening and diagnostic tests}

Details of the MS/MS screening methodology and diagnostic confirmation used in the included studies are provided in Additional file 1: supplement 6. In brief, two studies used commercially available MS/ MS assays [18, 23] while all others used non-kit methods with derivatisation of SUAC to its hydrazine $[12,14,17,19-22,24]$. MS/MS analysis of SUAC-hydrazone [12, 14, 19, 20, 24] or SUAC-hydrazone butyl ester [17, 21-23] was performed; the MS/MS methodology used was not reported by Lund et al. [18]. The SUAC cut-off values used in the 10 studies ranged from $1.29 \mu \mathrm{mol} / \mathrm{l}$ [23] to $10 \mu \mathrm{mol} / \mathrm{l}$ [19]. No two studies used the same cut-off value.

A range of approaches were reported for defining the reference standard. For individuals who screened positive or were used as cases these were: clinical diagnosis [14], "pre-natal testing" [12], DNA analysis [17, 18], analysis of SUAC in urine [12, 18, 19], analysis of plasma amino acids [12, 18], "diagnostically confirmed in accordance with institutional guidelines" [23], and on the basis of "symptoms consistent with TYR1" [20]. The method of diagnosis was not reported by Dhillon and colleagues [21] and la Marca and colleagues [22]. Four studies used more than one diagnostic approach [12, 18-20]. For individuals who screened negative, no clinical follow-up or other reference standard was reported for all five experience reports [12, 17-20]. In the five case-control studies, two conducted secondtier testing (SUAC or 5-aminolevulinic acid dehydratase [ALAD]) in DBS with elevated tyrosine levels to identify healthy controls [23, 24], while the reference standard to confirm absence of TYR1 was unclear in the other three studies [14, 21, 22].

\section{Accuracy of screening tests}

The methods and thresholds used for screening, and diagnostic tests varied between studies. Results were considered positive when they exceeded the threshold as set in the individual study. Table 1 shows the test accuracy data on sensitivity, specificity, positive predictive value (PPV) and negative predictive value (NPV).

\section{Sensitivity and specificity}

It was not possible to calculate sensitivity and specificity for the studies reporting screening experiences due to a lack of follow-up of people who had screened negative. For the case-control studies, sensitivity was estimated to be $100 \%$ in each of the five studies, which included 29 cases in total $[14,17,21,23,24]$. Specificity was estimated to be $100 \%$ in four studies [14, 22-24]. This included 34,403 unaffected babies in total, 18,204 of which had an inadequate reference standard. Specificity could not be calculated for the study by Dhillon 


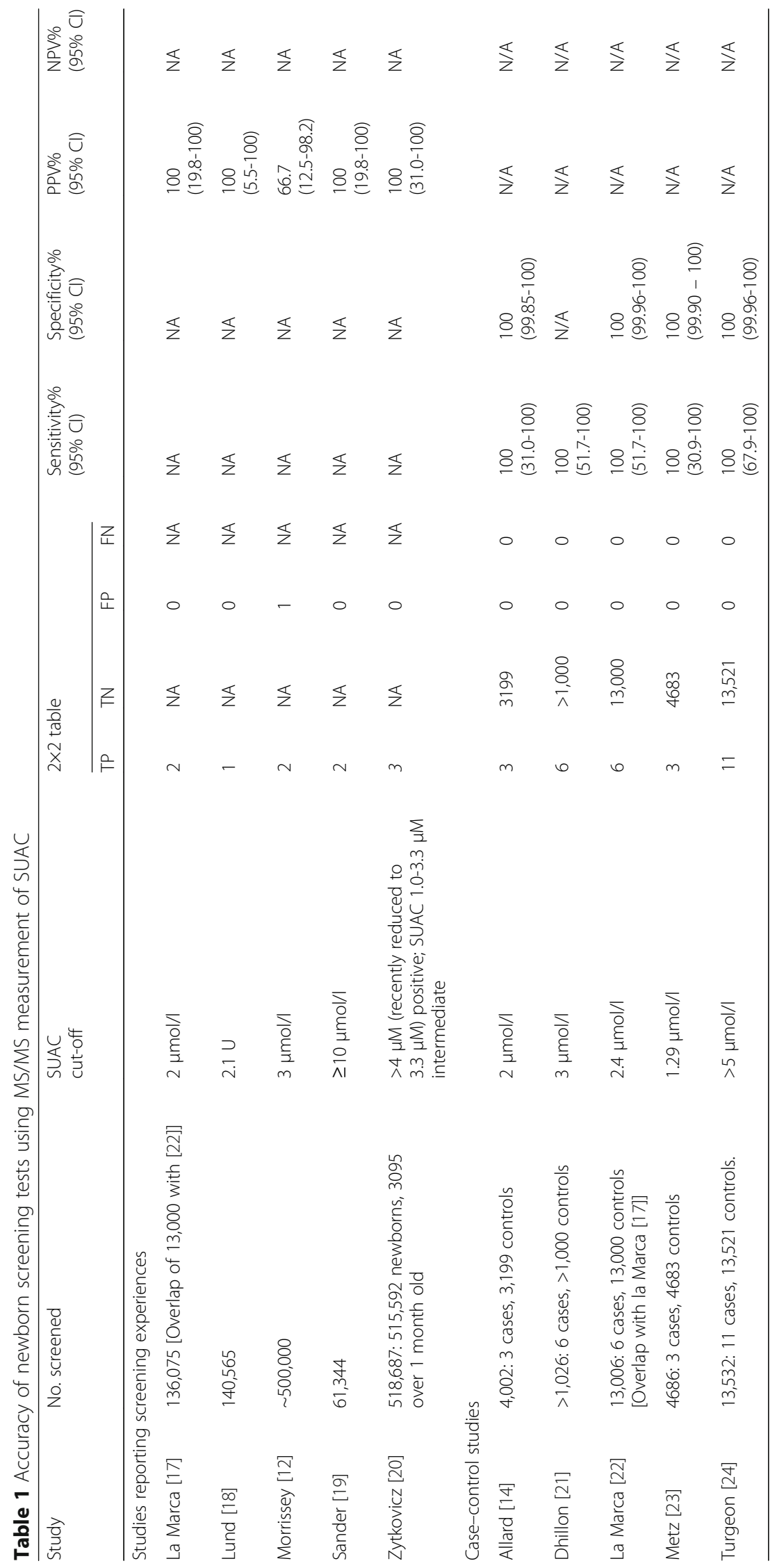


et al. [21] as they did not give a precise figure for the number of controls.

\section{Positive and negative predictive value}

In the studies reporting screening experiences, the PPV was $100 \%$ in four studies, with in total eight true positive cases and no false positive cases out of 856,671 people screened [17-20], and $66.7 \%$ in one study, with two true positive cases and one false positive case out of $\sim 500,000$ babies screened [12]. There were very wide confidence intervals due to the small number of cases. NPV could not be calculated due to a lack of follow-up of people who had screened negative. PPV and NPV could not be calculated from the case-control studies as these values are dependent on the prevalence of the disease in the population that is being tested.

\section{Discussion}

We examined the test accuracy of SUAC measurement in DBS using MS/MS to screen for TYR1 in newborns. Ten studies were identified which reported test accuracy data; five studies reporting screening experiences and five case-control studies. PPV in the studies reporting screening experiences ranged from $67 \%$ (two true positive cases and one false positive case out of $\sim 500,000$ babies screened) to $100 \%$ (eight true positive cases and no false positive cases out of 856,671 people screened). We were unable to calculate sensitivity, specificity, or negative predictive value in these studies due to a lack of follow-up of babies who screened negative. Case-control studies reported clear discrimination between SUAC levels of newborns with and without TYR1.

No consistent test accuracy metric was available. Papers reporting screening experiences suggested that using SUAC to screen for TYR1 resulted in no false negative results, and reported test sensitivity and specificity of up to $100 \%$. However, these conclusions were based on a lack of awareness of false negative results rather than following up babies who had screened negative. Without proper follow-up of the population who have been tested, for an appropriate amount of time, it is not possible to know if the absence of awareness of false negatives reflects an actual absence of false negative results.

While case-control studies showed no overlap in SUAC levels between newborns with and without TYR1, the cut-offs used varied between studies and were specified retrospectively, and the assessors were not blinded to the disease status, which can result in overestimation of test accuracy. The included case-control studies were also at high or unclear risk of differential verification bias as TYR1 cases and healthy controls received different reference standards, the reference standards used were not reported in sufficient detail to assess if their accuracy was comparable, or they were not reported at all.
The use of multiple reference standards across participants of a single study might have resulted in an overestimation of accuracies [25]. In addition, studies evaluating diagnostic tests in a diseased population and a separate healthy control group can overestimate the diagnostic performance compared with studies that use the index test in a clinical population covering the full range of patients without knowing their disease status [25].

Our understanding of the appropriateness of screening for TYR1 using SUAC is limited by heterogeneity in study design, the methods used for SUAC determination on DBS, and the SUAC cut-off values. For example, the SUAC cut-offs used in the screening test to identify possible cases of TYR1 ranged from $1.29 \mu \mathrm{mol} / \mathrm{l}$ [23] to $10 \mu \mathrm{mol} / \mathrm{l}$ [19]. Proficiency testing results for SUAC in dried blood spots have shown large differences among screening laboratories in SUAC recovery reflecting analytic biases, which might explain the wide variation in cut-off values of the studies in our review [26]. Differences in recovery could be explained by the method used (kit TMS vs. non-kit TMS; butyl ester derivatisation vs. non-derivatisation), DBS extraction strategy (freshly punched DBS, residual DBS or co-extraction of AA, AC, and SUAC, respectively), internal standard used $\left({ }^{13} \mathrm{C}\right.$-SUAC, 5,7-dioxooctanoic acid, or TMS kit internal standard), or the calibration strategy used (DBS calibrators, TMS internal standard/other liquid standard or kit internal standard only, respectively). Laboratories that measure low quantitative SUAC results usually used lower cut-off values to avoid misclassifications [26]. This highlights an important issue in how screening tests are evaluated. In this paper, we examine test accuracy, meaning the association between results from the test under investigation with the presence or absence of the target disease. However, the term 'accuracy' has multiple meanings. Within method validation (the process used to confirm that tests are suitable for their intended purpose), 'analytical' accuracy refers to the degree to which test results and the true value of the measured quantity agree and how reproducible and reliable the test is [27]. The analytical performance of the used SUAC assays has been described in some of the included studies. The recovery of SUAC was assessed in five studies by assaying DBS specimens enriched with predetermined (low to high) SUAC concentrations and was reported to be $51 \%$ [23], 72-80\% [19], 75-78\% [14], 75-86\% [21], and 97$100 \%$ [22] of the expected value, respectively. The quantification limit (the lowest amount of SUAC in a sample which can be reliably quantified) was reported in four studies and was $0.4 \mu \mathrm{mol} / \mathrm{l}$ [22], $0.5 \mu \mathrm{mol} / \mathrm{l}[19,23]$ and $1 \mu \mathrm{mol} / \mathrm{l}$ [14]. The calibration was reported to be linear up to $50 \mu \mathrm{mol} / \mathrm{l}$ [14], $100 \mu \mathrm{mol} / \mathrm{l}$ [19, 22, 24], $240 \mu \mathrm{mol} / \mathrm{l}$ [21], and $250 \mu \mathrm{mol} / \mathrm{l}$ [23], respectively. Precision (the 
ability to consistently reproduce a result when subsamples are taken from the same specimen) results were presented in seven studies with inter-assay coefficients of variation $(\mathrm{CV})$ at different SUAC concentrations of $10.0-12.2 \%$ [14], 7.1-8.5\% [21], 3.50-4.49\% [22], 5.8-13\% [19], $15.8-16.7 \%$ [24], $17.29-19.00 \%$ [23] and $30 \%$ in a pooled sample assay [20]. Taken together, the analytical performance of the screening tests used in the included studies was in agreement with previously reported proficiency testing outcomes [26, 28, 29], showing large between-laboratory differences in SUAC recoveries (mostly incomplete recoveries) depending on the method used and reproducible within-laboratory recoveries. There is need to harmonise quantitative results among laboratories. Despite differences among methods in SUAC recoveries (analytical bias), each method seems to have an acceptable precision and might therefore still be able (when using a cutoff value appropriate for the selected method) to reliably sort asymptomatic newborns into probable TYR1 cases and non-cases. De Jesus et al. [29] and Adam et al. [26] stress in their papers that bias in quantitative results can be tolerated if the screening test reliably sorts people into those who (probably) do have the disease of interest and those who (probably) don't. Any differences in the test accuracy between studies might be due to the timing of the test, the SUAC assay used, the cut-off used for classifying the disease status, use of repeat testing in samples with borderline SUAC levels, or variation in normal SUAC values in the tested newborn population.

Our review has a number of limitations. First, we were unable to synthesise our findings numerically due to incomplete $2 \times 2$ tables for reporting screening experiences, and heterogeneity in study design, the MS/MS method used, and the SUAC cut-off values. Second, we restricted our search to English language papers; non-Englishlanguage papers may be available and add further information. Third, we tailored the applicability questions for the QUADAS-2 in relation to the newborn screening in the UK. For example, in the UK newborn screening takes place five to eight days after birth, so studies in which samples were taken before or after this were rated as having high concerns regarding applicability. None of the studies we identified were conducted in the UK, and the usual time at which screening takes place varies by country; in many European countries newborn screening is conducted three days after birth. Therefore, the criteria for a high applicability concern might be different outside the UK.

While results from case-control studies are promising they are not definitive, as we know that case-control designs tend to overestimate test accuracy [25]. A research project using MS/MS measurement of SUAC from DBS with follow-up of screen-negatives for at least two years would considerably strengthen the test accuracy data. This could be achieved by following up one of the existing cohorts described in this review by searching hospital/primary care databases for cases of TYR1 that were identified symptomatically. While this approach would not provide a definitive answer, it would enable a measure of falsenegative cases that is currently missing from the literature.

\section{Conclusions}

MS/MS measurement of SUAC from DBS looks like a promising screening test for TYR1 but test accuracy from proof-of-concept studies should be confirmed in screening studies that include appropriate follow-up of screen-negatives.

\section{Additional file}

Additional file 1: Supplement 1 Search strategy for Ovid Medline (26th January 2016). Supplement 2 QUADAS-2 checklist. Supplement 3 Modified QUADAS-2 and guidance notes for tyrosinaemia type 1 screening. Supplement 4 Excluded studies $(n=66)$. Supplement 5 Risk of bias and applicability concerns summary: review authors' judgements about each domain for each included study. Supplement 6 Study characteristics and MS/MS screening methodology for Tyrosinemia type 1. (DOCX 78 kb)

\section{Abbreviations}

ALAD: 5-aminolevulinic acid dehydratase; CV: Coefficient of variation; DBS: Dried blood spot; MS/MS: Tandem mass spectrometry; NPV: Negative predictive value; PPV: Positive predictive value; QUADAS-2: Quality assessment tool for diagnostic accuracy studies 2; SUAC: Succinylacetone; TYR1: Tyrosinemia type 1

\section{Acknowledgements}

This research was commissioned by the UK National Screening Committee. Sian Taylor-Phillips, Aileen Clarke, Chris Stinton, and Hannah Fraser are supported by the NIHR CLAHRC West Midlands initiative. The views expressed in this publication are those of the authors and not necessarily those of the NHS, the National Institute for Health Research, the UK National Screening Committee, Public Health England or the Department of Health. Any errors are the responsibility of the authors.

Funding

This research was commissioned by the UK National Screening Committee.

\section{Availability of data and materials}

The datasets supporting the conclusions of this article are included within the article and its additional files.

\section{Authors' contributions}

CS conducted all aspects of the review and co-wrote the paper with JG. JG conducted all aspects of the review and co-wrote the paper with CS. KF assisted in the conduct of the review, provided methodological advice on systematic review methodology and commented on draft and final versions of the paper. AC undertook project planning and collaborated on research design. She read and commented on first and final drafts of the paper. SJ developed and conducted the literature searches, managed references and helped in obtaining full text references. HF helped in obtaining full text references, conducted data extraction and quality appraisal, commented on draft and final versions of the paper. PS assisted with funding and design, and commented on draft and final versions of the paper. STP secured the funding, undertook project planning and research design, co-ordinated the review process, and commented on draft and final versions of the paper. All members of the team contributed to the development of the protocol. All authors read and approved the final manuscript. 


\section{Competing interests}

The authors declare that they have no competing interests.

\section{Consent for publication}

Not applicable.

\section{Ethics approval and consent to participate}

Not applicable.

\section{Author details}

'Warwick Medical School, University of Warwick, Coventry CV4 7AL, England.

${ }^{2}$ Warwick Library, University of Warwick, Coventry CV4 7AL, England.

Received: 22 December 2016 Accepted: 23 February 2017

Published online: 09 March 2017

\section{References}

1. Lindblad B, Lindstedt S, Steen G. Enzymic defects in hereditary Tyrosinemia. Proc Natl Acad Sci U S A. 1977;74(10):4641-5.

2. de Laet C, Dionisi-Vici C, Leonard JV, McKiernan P, Mitchell G, Monti L, de Baulny HO, Pintos-Morell G, Spiekerkoetter U: Recommendations for the management of tyrosinaemia type 1. Orphanet Journal of Rare Diseases 2013, 8

3. Van Spronsen FJ, Thomasse Y, Smit GPA, Leonard JV, Clayton PT, Fidler V, Berger R, Heymans HSA. Hereditary tyrosinemia type 1: a new clinical classification with difference in prognosis on dietary treatment. Hepatology. 1994;20(5):1187-91.

4. Bartlett DC, Lloyd C, McKiernan PJ, Newsome PN. Early nitisinone treatment reduces the need for liver transplantation in children with tyrosinaemia type 1 and improves post-transplant renal function. J Inherit Metab Dis. 2014;37(5):745-52.

5. Larochelle J, Alvarez F, Bussieres J-F, Chevalier I, Dallaire L, Dubois J, Faucher F, Fenyves D, Goodyer P, Grenier A, et al. Effect of nitisinone (NTBC) treatment on the clinical course of hepatorenal tyrosinemia in Quebec. Mol Genet Metab. 2012;107(1-2):49-54.

6. McKiernan PJ, Preece MA, Chakrapani A. Outcome of children with hereditary tyrosinaemia following newborn screening. Arch Dis Child. 2015;100(8):738-41.

7. Debraekeleer M, Larochelle J. Genetic epidemiology of hereditary tyrosinemia in Quebec and in Saguenay-Lac-St-Jean. Am J Hum Genet. 1990:47(2):302-7.

8. Therrell BL, Lloyd-Puryear MA, Camp KM, Mann MY. Inborn errors of metabolism identified via newborn screening: Ten-year incidence data and costs of nutritional interventions for research agenda planning. Mol Genet Metab. 2014;113(1-2):14-26.

9. Hutchesson ACJ, Hall SK, Preece MA, Green A. Screening for tyrosinaemia type I. Arch Dis Child. 1996:74(3):F191-4.

10. Hadj-Taieb S, Nasrallah F, Hammami MB, Elasmi M, Sanhaji H, Moncef F, Kaabachi N. Aminoacidopathies and organic acidurias in Tunisia: a retrospective survey over 23 years. La Tunisie Medicale. 2012;90(3):258-61.

11. Goulden KJ, Moss MA, Cole DEC, Tithecott GA, Crocker JFS. Pitfalls in the initial diagnosis of tyrosinemia - 3 case reports and a review of the literature. Clin Biochem. 1987;20(3):207-12

12. Morrissey MA, Sunny S, Fahim A, Lubowski C, Caggana M. Newborn screening for Tyr-l: Two years' experience of the New York State program. Mol Genet Metab. 2011;103(2):191-2.

13. Wilcken B, Haas M, Joy P, Wiley V, Bowling F, Carpenter K, Christodoulou J, Cowley D, Ellaway C, Fletcher J, et al. Expanded newborn screening: outcome in screened and unscreened patients at Age 6 years. Pediatrics. 2009;124(2):E241-8.

14. Allard P, Grenier A, Korson MS, Zytkovicz TH. Newborn screening for hepatorenal tyrosinemia by tandem mass spectrometry: analysis of succinylacetone extracted from dried blood spots. Clin Biochem. 2004;37(11):1010-5.

15. Screening for Tyrosinaemia I. External review against programme appraisal criteria for the UK National Screening Committee (UK NSC). [legacy. screening.nhs.uk/policydb_download.php?doc $=474$ : NSC UK National Screening Committee]. Accessed 14 Mar 2016.

16. Whiting PF, Rutjes AWS, Westwood ME, Mallett S, Deeks JJ, Reitsma JB, Leeflang MMG, Sterne JAC, Bossuyt PMM, Grp Q. QUADAS-2: a revised tool for the quality assessment of diagnostic accuracy studies. Ann Intern Med. 2011:155(8):529-U104

17. la Marca G, Malvagia S, Pasquini E, Cavicchi C, Morrone A, Ciani F, Funghini S, Villanelli F, Zammarchi E, Guerrini R. Newborn screening for tyrosinemia type I: further evidence that succinylacetone determination on blood spot is essential. JIMD Reports. 2011;1:107-9.

18. Lund AM, Hougaard DM, Simonsen $H$, Andresen BS, Christensen M, Duno M, Skogstrand K, Olsen RKJ, Jensen UG, Cohen A, et al. Biochemical screening of 504,049 newborns in Denmark, the Faroe Islands and Greenland - Experience and development of a routine program for expanded newborn screening. Mol Genet Metab. 2012;107(3):281-93.

19. Sander J, Janzen N, Peter M, Sander S, Steuerwald U, Holtkamp U, Schwahn B, Mayatepek E, Trefz FK, Das AM. Newborn screening for Hepatorenal tyrosinemia: Tandem mass spectrometric quantification of succinylacetone. Clin Chem. 2006:52(3):482-7.

20. Zytkovicz TH, Sahai I, Rush A, Odewale A, Johnson D, Fitzgerald E, Britton D, Eaton RB. Newborn screening for hepatorenal tyrosinemia-l by tandem mass spectrometry using pooled samples: A four-year summary by the New England newborn screening program. Clin Biochem. 2013;46(7-8):681-4.

21. Dhillon KS, Bhandal AS, Aznar CP, Lorey FW, Neogi P. Improved tandem mass spectrometry (MS/MS) derivatized method for the detection of tyrosinemia type I, amino acids and acylcarnitine disorders using a single extraction process. Clin Chim Acta. 2011;412(11-12):873-9.

22. Ia Marca G, Malvagia S, Pasquini E, Innocenti M, Fernandez MR, Donati MA Zammarchi E. The inclusion of succinylacetone as marker for tyrosinemia type I in expanded newborn screening programs. Rapid Commun Mass Spectrom. 2008;22(6):812-8.

23. Metz TF, Mechtler TP, Merk M, Gottschalk A, Lukacin R, Herkner KR, Kasper DC. Evaluation of a novel, commercially available mass spectrometry kit for newborn screening including succinylacetone without hydrazine. Clin Chim Acta. 2012;413(15-16):1259-64.

24. Turgeon C, Magera MJ, Allard P, Tortorelli S, Gavrilov D, Oglesbee D, Raymond K, Rinaldo P, Matern D. Combined newborn screening for succinylacetone, amino acids, and acylcarnitines in dried blood spots. Clin Chem. 2008;54(4):657-64.

25. Lijmer JG, Mol BW, Heisterkamp S, Bonsel GJ, Prins MH, van der Meulen JHP, Bossuyt PMM. Empirical evidence of design-related bias in studies of diagnostic tests. JAMA. 1999;282(11):1061-6.

26. Adam B, Hall E, Meredith N, Lim T, Haynes C, De Jesus V, Hannon W. Performance of succinylacetone assays and their associated proficiency testing outcomes. Clin Biochem. 2012;45(18):1658-63.

27. International Conference on Harmonization: Q2A. Validation of Analytical Procedures. Federal Register. 1995;(60):11260-62.

28. Adam B, Lim T, Hall E, Hannon W. Preliminary proficiency testing results for succinylacetone in dried blood spots for newborn screening for tyrosinemia type I. Clin Chem. 2009:55(12):2207-13.

29. De Jesus VR, Adam BW, Mandel D, Cuthbert CD, Matern D. Succinylacetone as primary marker to detect tyrosinemia type I in newborns and its measurement by newborn screening programs. Mol Genet Metab. 2014:113(1-2):67-75.

\section{Submit your next manuscript to BioMed Central and we will help you at every step:}

- We accept pre-submission inquiries

- Our selector tool helps you to find the most relevant journal

- We provide round the clock customer support

- Convenient online submission

- Thorough peer review

- Inclusion in PubMed and all major indexing services

- Maximum visibility for your research

Submit your manuscript at www.biomedcentral.com/submit
C) Biomed Central 